\title{
From Home to Definitive Care for Critically Ill Children: Barriers and Solutions
}

\section{Andrew C. Argent, MB, BCh, MD, FCPaeds, FRCPCH}

\author{
Address \\ Paediatric Intensive Care Unit, Red Cross War Memorial Children's Hospital and \\ School of Child and Adolescent Health, University of Cape Town, Rondebosch, \\ Cape Town, 7700, South Africa \\ Email: Andrew.argent@uct.ac.za
}

Published online: 19 April 2015

(C) Springer International Publishing AG 2015

This article is part of the Topical Collection on Global Health

Keywords Children - Critical care - Pathways to care - Emergency care - Intensive care

\section{Opinion statement}

Optimal outcomes for children with a life-threatening illness or injury depend on early access to care; rapid recognition of the severity of illness with early initiation of appropriate and effective therapy; and optimized and rapid transport to units (such as intensive care units where definitive care can be provided). The details of this process may vary substantially depending on both the context and the underlying disease process which adds to the complexity of providing appropriate systems for children. In addition healthcare systems are frequently designed around the needs of adult patients and optimization of paediatric outcomes requires specific focus on the needs of children. Unfortunately, there are currently multiple barriers to such care, particularly in poorer parts of the world (where the bulk of critically ill or injured children are to be found). Improvement of this situation depends on a process which analyses the pathway to care, identifies challenges and problems in that pathway and provides focussed and system orientated interventions to improve care at all levels and throughout the system.

\section{Introduction}

Although the terms intensive care and critical care are often used interchangeably, it may be useful to use the term 'critical care' to describe the care required by a child with a life-threatening injury or illness (from first presentation to health services through to discharge home), while the term 'intensive care' could be limited to care delivered within the confines of the intensive care unit [1].
The exact time of onset of a critical illness or injury may be difficult to define depending on the specific context. The child involved in a motor vehicle accident has a clear time of onset of the injury, and it is usually clear immediately that the child requires medical care. By contrast, a child who has acquired a severe adenoviral infection may start out with relatively mild symptoms which may progress over a period of days despite the 
provision of appropriate medical intervention. Children who eventually present with life-threatening sepsis have often been unwell with mild symptoms such as diarrhoea over several days. In addition a significant proportion of children who develop acutely life-threatening illnesses have underlying conditions or preceding symptoms that have been present for some time prior to the onset of the critical illness.

The progression of life-threatening illness may also vary considerably from the rapid and dramatic progression of meningococcal septicaemia to the gradual and insidious progression of tuberculous meningitis. Finally, the definitive care required for a critically ill child may vary considerably in cost, complexity and availability.
The route from home to definitive care for critically ill children may be complex (Fig. 1) and involves an interaction between the child's family, the child and the health services (Table 1). The initial phases of this route are likely to be driven by the caregiver while the later stages of this process will be largely in the control of the health services. In most countries, the route to care is complicated by the fact that children with lifethreatening illness or injury are more likely to come from poorer communities (often cultural or language minorities; have limited access to private transport (and after hours, public transport may be limited); may live in environments where movement after dark may be relatively dangerous and where access to $24 \mathrm{~h}$ healthcare services may be extremely limited.

\section{Pathways to care}

In 1994, Thaddeus and Maine [2] considered the barriers to effective management of women with obstetrical problems using the '3-delays' framework. The framework delineated the pathway to care in terms of (1) deciding to seek emergency care, (2) identifying and reaching an appropriate facility and (3) receiving adequate and appropriate care at the facility. More recently, Binder et al. [3] have used that framework to highlight the complexities of that pathway, focussing on how even in high income areas; patients from low income backgrounds may have compromised access to effective patient care. They highlighted differences and similarities between high and low income settings and the impact of the different expectations of both healthcare seekers and healthcare providers. Likewise, Samuelsen et al. [4] reviewed the experience of mothers who had sought help in rural Tanzania, showing that confusion over issues such as payment, referral routes and communication adversely affected access to care - particularly for the poorest families.

\section{Access to critical care for children}

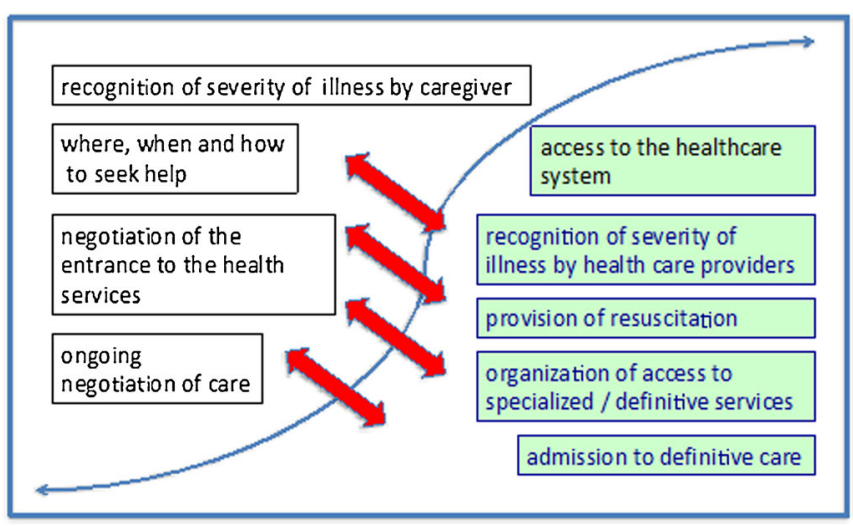

Fig. 1. There may be a complex interaction between parents and healthcare providers as parents seek care for their sick children. Communication may be problematic at multiple levels (language, social class, financial resources and clinical skills). The interaction between providers and parents may be affected by previous experience and by expectations on both sides. However, the further into the system that the child progresses, the higher the control exerted by the healthcare providers. 


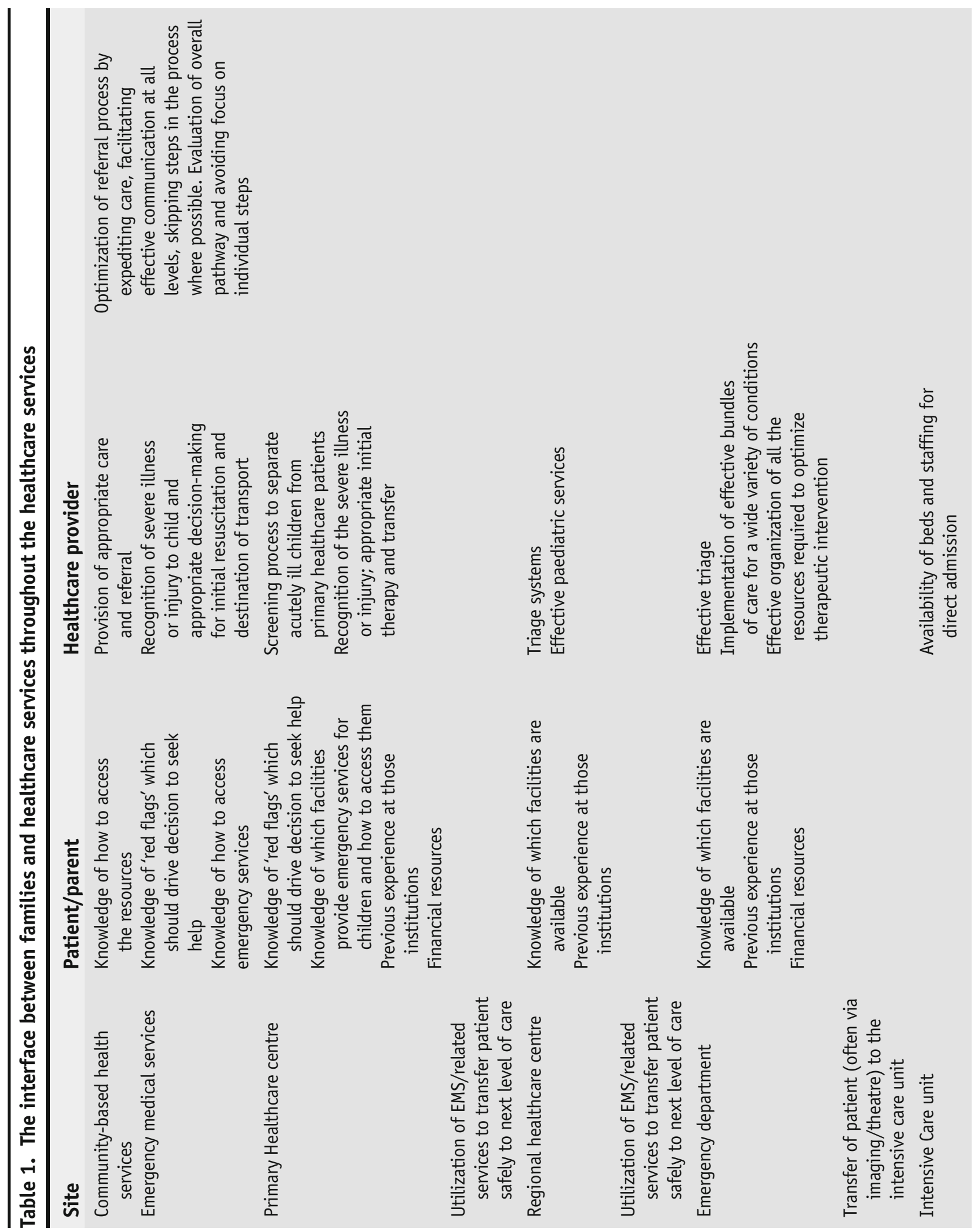




\section{Caregiver decisions around access to care}

Many processes to improve healthcare services have focussed on improvement of supply of healthcare services. However, there is some evidence that it may be at least as important to focus on the demand side [5], i.e. focussing on the factors that alert people to the need to seek healthcare and the factors that affect their behaviour in choosing where to seek that healthcare.

Caregiver recognition of serious illness or injury

The barriers to healthcare seeking and healthcare access were recently rated as one of the most important research questions to be addressed in the programme to reduce worldwide mortality from pneumonia in children [6]-despite the fact that currently, there is relatively little funded research in this area.

The initial step is the recognition by the caregiver of the child that something is wrong and requires medical attention. A significant proportion of childhood deaths worldwide are the consequence of infections such as pneumonia, malaria and diarrhoea, all of which are imminently amenable to effective therapy, particularly if provided early in the development of the condition. A recent meta-analysis of studies on recognition of severe paediatric illness in lower to middle income countries [7•] showed that the sensitivity and specificity of recognition of childhood conditions by caregivers was low with median sensitivity of 36,46 and $37 \%$ and median specificity of 96,67 and $58 \%$ for malaria, pneumonia and diarrhoea, respectively. It could be argued that it is not critical whether parents recognize the specific illness, but simply that there is a need for intervention. It is interesting that in Nairobi, while diarrhoea was recognized as a problem, coughing was perceived as being less important [8]. In Malawi, healthcare seeking for acute bacterial meningitis was often delayed while parents attempted some malaria therapy at home [9•], highlighting the complexity of recognizing severe illness when multiple infectious diseases are common. A recent study of newly delivery mothers in Uganda 
demonstrated that only 58.2 and $14.8 \%$ could identify one or two neonatal 'danger signs', respectively [10•].

Care seeking behaviour is certainly affected by maternal education [11, 12], and addressing this is an issue that extends beyond health service provision. There is data from Zambia showing that education programmes for communities regarding warning signs of severe illness may improve healthcare seeking behaviour from parents [13], although the changes in behaviour were not dramatic and took a period of years. There is also evidence that training of community healthcare workers may improve referral to healthcare services of sick infants [14].

There is a population of children with chronic illness who may have a substantially increased risk of acute illness requiring intensive care admission or at least critical care intervention $[15,16]$. Particular focus on providing 'red flag' warning information and defining clear pathways to care for these children may have a significant impact on reducing time to PICU admission.

Once the decision to seek help has been made; other issues become important as the caregiver considers where to seek help (this will be affected by issues such as which healthcare resources are available-particularly at that time of day); how they can be accessed and what transport is available; what previous experience does the caregiver have of those resources; what resources are required and available to access that healthcare.

Clearly, access to healthcare resources is an important factor in caregiver behaviours in seeking help for their children. In Brazil, an increase in healthcare workers was directly associated with a reduction in neonatal mortality [17]. A study of healthcare seeking behaviour of parents in slums of Nairobi Kenya [8] showed that financial constraints were the largest factor affecting healthcare seeking (similarly in Tanzania [4]). Removal of healthcare fees for under-5 children may have a significant impact on healthcare seeking behaviour [18], although this does not overcome limitations related to geographical access. However, seeking healthcare may also have out of pocket expenses (transport costs, loss of income, etc.), and the decision to spend those scarce resources may be particularly difficult for families who find it difficult to assess the severity of illness of the child [19].

Transport to care may be a major consideration for people in rural areas. Even within urban areas, after hours healthcare services (office hours usually only extend for approximately $25 \%$ of the week) may be much further from home than primary healthcare services and transport to those services may be extremely difficult for people with access to personal vehicles.

The parental decision to seek care at different levels of the health system may be affected by multiple factors. Parents in Kenya refused referral to regional hospitals because they had a perception that the hospitals were unable to provide more care then the local primary healthcare services [20]

It is also important to consider the organizational structure of emergency care services for children. In most situations, emergency services for children are provided within the context of health services that are primarily focussed on the provision of either primary care for children or emergency services for adults.

There is substantial evidence that emergency care for children is associated with improved outcomes when provided in the context of specialized paediatric 


\section{Health system issues}

services. This is offset by some data suggesting that optimization of patient care is also related to the patient load in that service.

\section{Recognition of critical illness or injury by healthcare workers}

A recent systematic review showed that frontline healthcare workers in LMIC were able to diagnose possible bacterial infections in children with reasonable sensitivity and specificity $[21 \bullet]$.

Recognition of critical illness in infants and children who present to primary healthcare facilities may be particular challenging in the context of large number of relatively well children. In that context (where the vast majority of children are not critically ill), it is probably not productive to employ triage systems. Aside from that context, recognition of the early stages of critical illness may be extremely challenging. In a review of patients who died from severe meningococcal disease, Thompson et al. [22] concluded that the best predictors of outcome in this population were the doctors 'gut feel' and the conviction of the mother that this illness was different. In addition, healthcare workers in the primary care setting may see critically ill children very infrequently and thus have difficulty in both recognition and management of these patients.

Within emergency departments and hospitals, a wide variety of triage [23, $24,25 \bullet, 26]$ or paediatric early warning systems (PEWS) [27] have been implemented in an attempt to ensure that sick children are recognized and prioritized for management. Some of these systems have identified critically ill children by thresholds on particular vital signs; others have used scores related to several parameters, while yet others have identified certain groups of patients as being high risk. Some institutions have focussed on processes to improve documentation of and response to abnormal vital signs in children [28•]. The simple utilization of a paediatric early warning system on its own is unlikely to have any impact on patient care. Review of reports of the introduction of PEWS has highlighted the process of implementation including staff training; establishing of rapid response teams, etc.

Even within hospital wards, there is a need to develop systems to identify children who are deteriorating and require intervention.

\section{Provision of appropriate care}

\section{Community healthcare workers}

Bhutta et al. [29] have demonstrated that provision of simple antibiotics to village/community healthcare workers may substantially decrease the mortality of infants and children with severe infection. A number of innovative programmes have provided emergency and first aid training to villagers [30, 31] and or commercial drivers [32] as a means of ensuring that adults and children receive some appropriate care at the time of injury with some dramatic results

\section{Emergency medical services (EMS)}

The challenges of emergency medical systems vary across the world. In most of the world, the emergency services are unable to provide adequate care and one 
is left with a situation where the emergency transport of critically ill patients may be rudimentary [33]

The efficiency of emergency medical services in responding to calls to critically ill or injured children may differ substantially. This may relate to the resources available (such as personnel or vehicles), but it also may relate to processes such as those required to prioritize calls and dispatch vehicles with appropriately trained personnel to the site of the incident.

The geography of the region may have a substantial impact on the availability of emergency medical services. In general (despite traffic), it is much easier to provide emergency services to urban areas with established transport networks than it is to provide services either to rural areas or to peri-urban areas with informal housing and inadequate infrastructure.

Shah et al. [34] have demonstrated that the utilization of emergency medical services (EMS) services for children is substantially lower than that of adults, but children arriving in emergency departments via the EMS are more likely to have injuries, poisoning or other high acuity illness than patients arriving by other means.

In the richer countries of the world, transport services to move critically ill children between institutions and to intensive care units have developed sophisticated networks, while these are less developed in poorer countries [35]. In Malaysia, development of a referral network with patient transport was associated with improved outcomes [36, 37].

\section{District and regional hospitals}

There is a significant body of evidence to show that district and regional hospitals in poorer countries may be failing to provide the quality of care that is required for acutely ill children [20, 38, 39].

A detailed study showed that it was possible to achieve improvements in many aspects of quality of care in district hospitals using a focussed educational intervention [40•]

\section{Emergency departments}

In many settings, emergency departments have struggled with defining their roles. In providing ready access to care for those who need emergency care, they have run the risk of being overwhelmed by patients who are not perceived as requiring emergency care, and many reports from emergency departments are as much focussed on 'inappropriate' patients as they are on appropriate patients.

Even in richer countries of the world, there are concerns about the access to emergency care that is provided to children [41].

In general, the efficacy of care relates to the range of services that are offered [42]. It is clearly easier to ensure that appropriate care occurs for common conditions. However, there is data suggesting that even in well-established and equipped emergency departments, it is difficult to achieve compliance of $>30 \%$ with relatively simple bundles of care such as the Pediatric Advanced Life Support recommendations for acute care for paediatric sepsis [43].

While countries such as the USA have highly developed trauma systems, and more than $80 \%$ of adults will have access to sophisticated trauma services within an hour of injury, fewer children have access and in fact, some 17.4 million children in the USA do not have access to a paediatric trauma service within $1 \mathrm{~h}$ of injury [44]. Although not definitive, there is evidence that 
outcomes for paediatric trauma victims are better at dedicated paediatric trauma centres [45].

In many parts of the world, children with trauma are managed by adult emergency services, and this may not be entirely in their best interests. In Malawi, Molyneux et al. showed that the reorganization of paediatric emergency services (which included ensuring that paediatric trauma was seen in the paediatric emergency department) was followed by a substantial drop in child mortality in the hospital [46].

\section{Care of the critically ill in hospitals}

Even in tertiary hospitals, it may be challenging to care for critically ill children. Clearly, paediatric hospitals and paediatric healthcare systems, for trauma and other illness, have better outcomes for children.

A particular challenge has been the development of 'high care' services for children who are perhaps not that sick that they require intensive care but who do need monitoring that is more focussed than general wards can provide [47•]

Intensive care access

Limited intensive care access is the reality for most of the world. Even where intensive care access is available, but delayed, there is evidence that delays in admission are associated with increased need for advanced respiratory support and longer duration of ventilation [48]. That builds on the work [2] showing that delays in access to maternal care were associated with increased mortality and morbidity.

In many parts of the world, intensive care for children is only available in the context of neonatal or adult intensive care units and there is evidence that outcomes for children in these units may be substantially worse than what would be expected for specialized paediatric intensive care units [49]

There is evidence to support regionalization of paediatric intensive care services, to ensure that critically ill children are cared for in specialized paediatric units which have adequate patient turnover to enable a high level of experience and current expertise in the units. A consequence of regionalization is the demand for specialized transport services for critically ill children [50•], and there is evidence that the use of specialized transport services is associated with improved outcomes [35, 51-53]. Those transport teams may also be able to assist with stabilization of critically ill children at regional and district hospitals [54].

\section{What may delay care?}

There is limited data available internationally on the time taken from first contact with healthcare services through to definitive care for that specific condition. Studies in the USA on access to critical care for adults [55•] have highlighted the fact that barriers to effective critical care may exist at multiple stages all the way from community issues through to organization of tertiary healthcare services.

A number of authors have reviewed care throughout a health system in relation to specific conditions $[56,57]$ particularly related to sepsis. In each case, 
the authors have identified multiple steps in the healthcare process that have contributed to poor outcomes. In London, a particular intensive care unit focussed on the management of severe meningococcal sepsis in children. They were able to identify failures in care that were related to worse outcomes in children with meningococcaemia [56]. In particular, departures from recommended protocols were problematic and potentially amenable to intervention using education together with improved supervision by senior medical staff. In Brazil [58 $]$, implementation of multifaceted educational programmes regarding the management of adult sepsis were associated with significant improvement in compliance with recommended protocols and that in turn was associated with a drop in overall mortality and adverse outcomes.

Systematic review processes have been useful tools by which to identify problems in the health services as regards the management of critically ill children $[59,60 \bullet$. Ironically, the societies with the highest number of paediatric deaths have the lowest resources available to undertake detailed investigations of why those deaths happened. However, a focus on understanding systems and how they could be optimized is likely to pay dividends in most settings.

In the discussion above, I have focussed on the provision of acute care to critically ill children under normal conditions. Recently, a number of authors have addressed the question of provision of care for critically ill children under 'disaster' or surge conditions and it can be seen that this is potentially an even greater challenge [61-63]. Throughout the world, paediatric emergency and critical care services have much less 'surge capacity' than adult services.

\section{Implementation of care and quality of care}

In 2004, Angus et al. [64], in writing about caring for critically ill adults, pointed out that improvements in outcome would depend on education (not only of healthcare workers); organizational guidelines; performance reporting (which is closely linked to the establishment of both guidelines and regulations); incentives (both financial and socio-cultural); regulations and legal requirements; and healthcare reorganization.

Education of healthcare workers in the care of the critically ill can take place through a wide variety of routes including the conventional formal healthcare education, the use of short course such as the paediatric life support course and ongoing in-service training [65] and the use of innovative education processes [66•]. A particular challenge of these educational processes is the need for a multifaceted approach, with focussed intervention over a period of time [28•]

Fundamental to the improvement process is definition of what is acceptable and appropriate care [67]. Some of the challenges of this process include the development of measures of quality of acute care for specific conditions in particular areas [68•]. This has been done in some conditions such as severe sepsis, and it is sobering that in relatively well equipped institutions, compliance with recommendations for management of severe sepsis has been of the order of $30 \%$ [43]. However, in those studies, compliance has been associated with either better outcomes or at least reduction in hospital stay

Most quality improvement processes are focussed on individual departments within individual systems, but there is a need to review the overall 


\section{Conclusions}

pathway to care. In the United Kingdom, there have been calls to monitor among other things the time taken from first presentation of children through to definitive treatment [69] as a fundamental measure of quality of care for children.

One of the particular challenges of paediatric critical care is the range of conditions that are seen but in relatively (compared to adults) low numbers of patients. Thus, pathways to care will have to be developed around the needs of children (with associated expertise), rather than around the treatment needs of specific conditions as may be the case in adult critical care.

Optimal care for critically ill or injured children depends on the development of a seamless continuum of care including caregivers, community health services, and healthcare services at all levels. While it is possible to identify a wide range of interventions at multiple steps which may all play a role in the improvement of overall care, there is a need to consider the overall pathway from home to intensive care (or other definitive medical services) and to address the system in its entirety.

Optimization of critical care will require the co-operation of healthcare professionals at all levels but will ultimately require the teamwork of all roleplayers including managers and those that control the personnel and financial resources available for healthcare in general and for paediatric healthcare in specific.

\section{Compliance with Ethics Guidelines}

\section{Conflict of Interest}

Andrew C Argent declares that he has no conflict of interest.

Human and Animal Rights and Informed Consent

This article does not contain any studies with human or animal subjects performed by any of the authors.

\section{References and Recommended Reading}

Papers of particular interest, published recently, have been

highlighted as:

- Of importance

1. Kissoon N, Argent A, Devictor D, Madden MA, Singhi $\mathrm{S}$, van der Voort E, et al. World Federation of Pediatric Intensive and Critical Care Societies-its global agenda. Pediatr Crit Care Med. 2009;10(5):597-600.

2. Thaddeus S, Maine D. Too far to walk: maternal mortality in context. Soc Sci Med. 1994;38(8):1091-110.
3. Binder P, Johnsdotter S, Essen B. Conceptualising the prevention of adverse obstetric outcomes among immigrants using the 'three delays' framework in a highincome context. Soc Sci Med. 2012;75(11):2028-36.

4. Samuelsen H, Tersbol BP, Mbuyita SS. Do health systems delay the treatment of poor children? A 
qualitative study of Child Deaths in Rural Tanzania. BMC Health Serv Res. 2013;13:67.

5. Ensor T, Cooper S. Overcoming barriers to health service access: influencing the demand side. Health Policy Plan. 2004;19(2):69-79.

6. Rudan I, El Arifeen S, Bhutta ZA, Black RE, Brooks A, Chan KY, et al. Setting research priorities to reduce global mortality from childhood pneumonia by 2015 . PLoS Med. 2011;8(9):e1001099.

7. $\quad$ Geldsetzer P, Williams TC, Kirolos A, Mitchell S, Ratcliffe LA, Kohli-Lynch MK, et al. The recognition of and care seeking behaviour for childhood illness in developing countries: a systematic review. PLoS One. 2014;9(4):e93427.

This systematic review focussed on studies of healthcare seeking for children with malaria, diarrhoea or pneumonia, in low and middle income countries. A useful overview of the current literature highlighting areas for future research.

8. Taffa N, Chepngeno G. Determinants of health care seeking for childhood illnesses in Nairobi slums. Trop Med Int Health TM IH. 2005;10(3):240-5.

9.• Desmond NA, Nyirenda D, Dube Q, Mallewa M, Molyneux E, Lalloo DG, et al. Recognising and treatment seeking for acute bacterial meningitis in adults and children in resource-poor settings: a qualitative study. PLoS One. 2013;8(7):e68163.

This study focussed on the recognition of acute bacterial meningitis in countries where diseases such as malaria are common. They highlight the need for educational programs to facilitate early recognition of symptoms related to meningitis.

10.• Sandberg J, Odberg Pettersson K, Asp G, Kabakyenga J, Agardh A. Inadequate knowledge of neonatal danger signs among recently delivered women in southwestern rural Uganda: a community survey. PLoS One. 2014;9(5):e97253.

This study highlighted the limited knowledge of neonatal danger signs among mothers of newborn infants in Uganda. There is clearly a need to improve education of mothers at this stage of contact with the health services.

11. Malhotra N, Upadhyay RP. Why are there delays in seeking treatment for childhood diarrhoea in India? Acta Paediatr. 2013;102(9):e413-8.

12. Omore R, O'Reilly CE, Williamson J, Moke F, Were V, Farag TH, et al. Health care-seeking behavior during childhood diarrheal illness: results of health care utilization and attitudes surveys of caretakers in western Kenya, 2007-2010. Am J Trop Med Hyg. 2013;89(1 Suppl):29-40.

13. Sasaki S, Fujino Y, Igarashi K, Tanabe N, Muleya CM, Suzuki H. Access to a health facility and care-seeking for danger signs in children: before and after a community-based intervention in Lusaka, Zambia. Trop Med Int Health TM IH. 2010;15(3):312-20.

14. Nsibande D, Doherty T, Ijumba P, Tomlinson M, Jackson D, Sanders D, et al. Assessment of the uptake of neonatal and young infant referrals by community health workers to public health facilities in an urban informal settlement, KwaZulu-Natal, South Africa. BMC Health Serv Res. 2013;13:47.
15. Dosa NP, Boeing NM, Ms N, Kanter RK. Excess risk of severe acute illness in children with chronic health conditions. Pediatrics. 2001;107(3):499-504.

16. Mestrovic J, Kardum G, Polic B, Mestrovic M, Markic J, Sustic A, et al. The influence of chronic health conditions on susceptibility to severe acute illness of children treated in PICU. Eur J Pediatr. 2006;165(8):526-9.

17. Sousa A, Dal Poz MR, Boschi-Pinto C. Reducing inequities in neonatal mortality through adequate supply of health workers: evidence from newborn health in Brazil. PLoS One. 2013;8(9):e74772.

18. Ridde V, Haddad S, Heinmuller R. Improving equity by removing healthcare fees for children in Burkina Faso. J Epidemiol Community Health. 2013;67(9):751-7.

19. Alvesson HM, Lindelow M, Khanthaphat B, Laflamme L. Shaping healthcare-seeking processes during fatal illness in resource-poor settings. A study in Lao PDR. BMC Health Serv Res. 2012;12:477.

20. English M, Esamai F, Wasunna A, Were F, Ogutu B, Wamae A, et al. Assessment of inpatient paediatric care in first referral level hospitals in 13 districts in Kenya. Lancet 2004;363(9425):1948-53.

21. Lee AC, Chandran A, Herbert HK, Kozuki N, Markell P, Shah $\mathrm{R}$, et al. Treatment of infections in young infants in low- and middle-income countries: a systematic review and meta-analysis of frontline health worker diagnosis and antibiotic access. PLoS Med. 2014;11(10):e1001741.

This study provided a systematic review of the literature regarding the treatment of infections in young infants by frontline healthcare workers in low and middle income countries.

22. Thompson MJ, Ninis N, Perera R, Mayon-White R, Phillips C, Bailey L, et al. Clinical recognition of meningococcal disease in children and adolescents. Lancet. 2006;367(9508):397-403.

23. Seiger N, Maconochie I, Oostenbrink R, Moll HA. Validity of different pediatric early warning scores in the emergency department. Pediatrics. 2013;132(4):e84150.

24. Twomey M, Cheema B, Buys H, Cohen K, de Sa A, Louw $\mathrm{P}$, et al. Vital signs for children at triage: a multicentre validation of the revised South African Triage Scale (SATS) for children. S Afr Med J. 2013;103(5):304-8.

25. Buys H, Muloiwa R, Westwood C, Richardson D, Cheema B, Westwood A. An adapted triage tool (ETAT) at Red Cross War Memorial Children's Hospital Medical Emergency Unit, Cape Town: an evaluation. S Afr Med J S. 2013;103(3):161-5.

A description of the development of a triage tool at a tertiary referral hospital in Cape Town, South Africa.

26. Wallis PA, Gottschalk SB, Wood D, Bruijns S, de Vries S, Balfour C. The Cape Triage Score-a triage system for South Africa. S Afr Med J. 2006;96(1):53-6.

27. Parshuram CS, Duncan HP, Joffe AR, Farrell CA, Lacroix JR, Middaugh KL, et al. Multicentre validation of the bedside paediatric early warning system score: a severity of illness score to detect evolving critical illness in hospitalised children. Crit Care. 2011;15(4):R184. 
28.• McKay H, Mitchell IA, Sinn K, Mugridge H, Lafferty T, Van Leuvan C, et al. Effect of a multifaceted intervention on documentation of vital signs and staff communication regarding deteriorating paediatric patients. J Paediatr Child Health. 2013;49(1):48-56.

The authors used documentation of vital signs as a means of assessing the impact of a program directed at early recognition of critically ill children.

29. Saghafinia M, Nafissi N, Asadollahi R. Effect of the rural rescue system on reducing the mortality rate of landmine victims: a prospective study in Ilam Province, Iran. Prehosp Disaster Med. 2009;24(2):126-9.

30. Murad MK, Husum H. Trained lay first responders reduce trauma mortality: a controlled study of rural trauma in Iraq. Prehosp Disaster Med. 2010;25(6):533-9.

31. Tiska MA, Adu-Ampofo M, Boakye G, Tuuli L, Mock $\mathrm{CN}$. A model of prehospital trauma training for lay persons devised in Africa. Emerg Med J EMJ. 2004;21(2):237-9.

32. Scribante J, Bhagwanjee S. National audit of critical care resources in South Africa-transfer of critically ill patients. S Afr Med J. 2007;97(12 Pt 3):1323-6.

33. Shah MN, Cushman JT, Davis CO, Bazarian JJ, Auinger $\mathrm{P}$, Friedman B. The epidemiology of emergency medical services use by children: an analysis of the National Hospital Ambulatory Medical Care Survey. Prehosp Emerg Care. 2008;12(3):269-76.

34. Hatherill M, Waggie Z, Reynolds L, Argent A. Transport of critically ill children in a resource-limited setting. Intensive Care Med. 2003;29(9):1547-54.

35. Goh AY, Abdel-Latif Mel A, Lum LC, Abu-Bakar MN. Outcome of children with different accessibility to tertiary pediatric intensive care in a developing country-a prospective cohort study. Intensive Care Med. 2003;29(1):97-102.

36. Goh AY, El-Amin Abdel-Latif M. Transport of critically ill children in a resource-limited setting: alternatives to a specialized retrieval team. Intensive Care Med. 2004;30(2):339.

37. English M, Esamai F, Wasunna A, Were F, Ogutu B, Wamae A, et al. Assessment of inpatient paediatric care in first referral level hospitals in 13 districts in Kenya. Lancet. 2004;363(9425):1948-53.

38. English M, Ntoburi S, Wagai J, Mbindyo P, Opiyo $\mathrm{N}$, Ayieko $\mathrm{P}$, et al. An intervention to improve paediatric and newborn care in Kenyan district hospitals: understanding the context. Implement Sci IS. 2009;4:42.

39. Gathara D, Opiyo N, Wagai J, Ntoburi S, Ayieko P, Opondo C, et al. Quality of hospital care for sick newborns and severely malnourished children in Kenya: a two-year descriptive study in 8 hospitals. BMC Health Serv Res. 2011;11:307.

40. Mwaniki P, Ayieko P, Todd J, English M. Assessment of paediatric inpatient care during a multifaceted quality improvement intervention in Kenyan district hospitals-use of prospectively collected case record data. BMC Health Serv Res. 2014;14:312.
This study provides some insight into the methodology that can be utilized to track the impact of multifacetted interventions for improvement of healthcare delivery.

41. Yamamoto LG. Access to optimal emergency care for children. Pediatrics. 2007;119(1):161-4.

42. Modig NA P. This is Lean: Resolving the Efficiency Paradox: Rheologica Publishing. 2012.

43. Paul R, Neuman MI, Monuteaux MC, Melendez E. Adherence to PALS Sepsis Guidelines and Hospital Length of Stay. Pediatrics. 2012;130(2):e273-80.

44. Carr BG, Nance ML. Access to pediatric trauma care: alignment of providers and health systems. Curr Opin Pediatr. 2010;22(3):326-31.

45. Petrosyan M, Guner YS, Emami CN, Ford HR. Disparities in the delivery of pediatric trauma care. J Traumatol. 2009;67(2 Suppl):S114-9.

46. Molyneux E, Ahmad S, Robertson A. Improved triage and emergency care for children reduces inpatient mortality in a resource-constrained setting. Bull World Health Organ. 2006;84(4):314-9.

47. Morris KP, Oppong R, Holdback N, Coast J. Defining criteria and resource use for high dependency care in children: an observational economic study. Arch Dis Child. 2014;99(7):652-8.

A very useful study focussing on the criteria for high dependency care, which is relatively poorly described in the literature.

48. O'Callaghan DJ, Jayia P, Vaughan-Huxley E, Gribbon M, Templeton M, Skipworth JR, et al. An observational study to determine the effect of delayed admission to the intensive care unit on patient outcome. Crit Care. 2012;16(5):R173.

49. Pearson G, Shann F, Barry P, Vyas J, Thomas D, Powell $\mathrm{C}$, et al. Should paediatric intensive care be centralised? Trent versus Victoria. Lancet. 1997;349(9060):1213-7.

50.• Thompson LM, Armfield NR, Slater A, Mattke C, Foster M, Smith AC. The availability, spatial accessibility, service utilisation and retrieval cost of paediatric intensive care services for children in rural, regional and remote Queensland: study protocol. BMC Health Serv Res. 2013;13:163.

This study protocol outlines how the authors will study the factors that affect access to critical care for children in rural areas of a part of Australia.

51. Ramnarayan P, Thiru K, Parslow RC, Harrison DA, Draper ES, Rowan KM. Effect of specialist retrieval teams on outcomes in children admitted to paediatric intensive care units in England and Wales: a retrospective cohort study. Lancet. 2010;376(9742):698-704.

52. Odetola FO, Davis MM, Cohn LM, Clark SJ. Interhospital transfer of critically ill and injured children: an evaluation of transfer patterns, resource utilization, and clinical outcomes. J Hosp Med. 2009;4(3):164-70.

53. Gregory CJ, Nasrollahzadeh F, Dharmar M, Parsapour K, Marcin JP. Comparison of critically ill and injured children transferred from referring hospitals versus inhouse admissions. Pediatrics. 2008;121(4):e906-11.

54. Lampariello S, Clement M, Aralihond AP, Lutman D, Montgomery MA, Petros AJ, et al. Stabilisation of critically ill children at the district general hospital prior to 
intensive care retrieval: a snapshot of current practice. Arch Dis Child. 2010;95(9):681-5.

55. Soto GJ, Martin GS, Gong MN. Healthcare disparities in critical illness. Crit Care Med. 2013;41(12):2784-93.

This study reviewed all the factors associated with healthcare disparities in critical illness in the United States of America, showing the multiple factors that were complexly associated with healthcare disparities.

56. Ninis N, Phillips C, Bailey L, Pollock JI, Nadel S, Britto $\mathrm{J}$, et al. The role of healthcare delivery in the outcome of meningococcal disease in children: case-control study of fatal and non-fatal cases. BMJ. 2005;330(7506):1475.

57. Launay E, Gras-Le Guen C, Martinot A, Assathiany R, Blanchais T, Mourdi N, et al. Suboptimal care in the initial management of children who died from severe bacterial infection: a population-based confidential inquiry. Pediatr Crit Care Med. 2010;11(4):469-74.

58. Noritomi DT, Ranzani OT, Monteiro MB, Ferreira EM, Santos SR, Leibel F, et al. Implementation of a multifaceted sepsis education program in an emerging country setting: clinical outcomes and cost-effectiveness in a long-term follow-up study. Intensive Care Med. 2014;40(2):182-91.

This study reports on the implementation of a multi-faceted educational program in a group of hospitals. The program focussed on the management of severe sepsis and was associated with improved compliance with protocols and improved outcomes.

59. Pearson GA, Ward-Platt M, Harnden A, Kelly D. Why children die: avoidable factors associated with child deaths. Arch Dis Child. 2011;96(10):927-31.

60. Sa'avu M, Duke T, Matai S. Improving paediatric and neonatal care in rural district hospitals in the highlands of Papua New Guinea: a quality improvement approach. Paediatr Int Child Health. 2014;34(2):75-83.

This study focussed on the provision of oxygen therapy in rural hospitals in Papua New Guinea. This is a key marker of quality of care and is a useful focus as an area for improvement and development.
61. Antommaria AH, Powell T, Miller JE, Christian MD. Ethical issues in pediatric emergency mass critical care. Pediatr Crit Care Med. 2011;12(6 Suppl):S163-8.

62. Burkle Jr FM, Argent AC, Kissoon N. The reality of pediatric emergency mass critical care in the developing world. Pediatr Crit Care Med. 2011;12(6 Suppl):S16979.

63. Gomersall CD, Tai DY, Loo S, Derrick JL, Goh MS, Buckley TA, et al. Expanding ICU facilities in an epidemic: recommendations based on experience from the SARS epidemic in Hong Kong and Singapore. Intensive Care Med. 2006;32(7):1004-13.

64. Angus DC, Black N. Improving care of the critically ill: institutional and health-care system approaches. Lancet. 2004;363(9417):1314-20.

65. Sutton RM, Niles D, Meaney PA, Aplenc R, French B, Abella BS, et al. Low-dose, high-frequency CPR training improves skill retention of in-hospital pediatric providers. Pediatrics. 2011;128(1):e145-51.

66. Frehywot S, Vovides Y, Talib Z, Mikhail N, Ross H, Wohltien $\mathrm{H}$, et al. E-learning in medical education in resource constrained low- and middle-income countries. Hum Resour Health. 2013;11(1):4.

This paper summarizes the electronic tools available for medical education in low and middle income countries, providing a basis for further research and development.

67. Molyneux E, Weber MW. Applying the right standards to improve hospital performance in Africa. Lancet. 2004;364(9445):1560-1.

68. Stang AS, Straus SE, Crotts J, Johnson DW, Guttmann A. Quality indicators for high acuity pediatric conditions. Pediatrics. 2013;132(4):752-62.

The authors report on a process for developing quality indicators for the management of high acuity pediatric conditions in the emergency department setting.

69. Mooney H. NHS must monitor time from presentation to treatment for all child patients, report says. BMJ. 2012;345:e5138. 